\title{
Role of ovarian sympathetic nerves and cholinergic local system during cold stress
}

\author{
Raul Riquelme1, Freddy Ruz¹, Artur Mayerhofer² and Hernán E Lara1 \\ ${ }^{1}$ Center for Neurobiochemical studies in Endocrine Diseases, Laboratory of Neurobiochemistry, Department of Biochemistry and Molecular Biology, \\ Faculty of Chemistry and Pharmaceutical Sciences, Universidad de Chile, Santiago, Chile \\ 2Biomedical Center Munich (BMC), Cell Biology, Anatomy III, Ludwig-Maximilians-Universität München, Planegg-Martinsried, Germany
}

Correspondence should be addressed to H E Lara: hlara@ciq.uchile.cl

\begin{abstract}
An increase in the sympathetic tone in the rat ovary induces a polycystic ovary (PCOSlike) phenotype. No information exists about its impact on fertility. In contrast, increased follicular development and improved fertility in rats were found after pharmacological inhibition of acetylcholinesterase, which increased intraovarian acetylcholine (ACh). Now, we studied the impact of sympathetic stress, followed by a recovery period without stress, on the cholinergic and noradrenergic systems of the rat ovary and on fertility. To activate ovarian sympathetic nerves, female Sprague-Dawley rats were exposed to cold stress $\left(4^{\circ} \mathrm{C} / 3 \mathrm{~h}\right.$ day for 28 days; first period), followed by a 28 -day period without cold stress (second period). No changes in estrous cyclicity during the first period was found. At the end of this period, ovarian levels of NA and ACh were increased. Morphometric analysis showed lower numbers of secondary and antral follicles, enhanced follicular atresia and fewer corpora lutea. Plasma progesterone was lower and testosterone was higher than that in controls. At end of the second period, ovarian ACh levels had returned to control levels, but NA levels remained elevated. The second period was also characterized by the presence of cystic follicles in the ovary, by elevated plasma testosterone and estradiol levels, while progesterone levels were decreased. Estrous cyclicity and ovulation during that period were irregular and fertility decreased. Thus, cold stress initially activated both ovarian noradrenergic and cholinergic system. After stress, the ovary did not fully recover and activation of the noradrenergic system persisted and correlated with cystic ovarian morphology and decreased fertility.
\end{abstract}
Key Words
- stress
- polycystic ovary
- acetylcholine
- fertility

\section{Introduction}

Growing evidence suggests that ovarian function is under the control of the autonomic nervous system, which works in parallel with the hypothalamic-pituitarygonadal axis (HPG axis) (Ojeda 2012, Uchida 2015, Pastelín et al. 2017). Sympathetic nerves originating in the celiac ganglia project to the ovarian follicles, principally to its theca layer, and its transmitter, noradrenalin (NA), then acts on beta-adrenergic receptors located in theca and granulosa cells (Aguado 2002, Lara et al. 2002, Manni et al. 2005, Rosas et al. 2018). Both neural and endocrine signals converge to regulate steroidogenesis and ovarian follicular development. The development of follicles in the ovary is a complex process starting from primordial follicles, which are recruited for maturation in a cyclical manner and progress to different stages of development. When follicles reach the preovulatory stage, they respond 
to LH, allowing the oocyte to be released, while the remaining cells are luteinized and form a major part of the corpus luteum (Havelock et al. 2004). If ovulation does not occur properly, follicles persist and become fluid-filled follicular cysts (McGee \& Hsueh 2000). These structures produce androgens and are associated with deregulation of ovarian function in pathologies such as polycystic ovary syndrome (PCOS).

Exposure of adult rats to cold stress increases sympathetic activity and NA levels in the ovary (Dorfman et al. 2003, Bernuci et al. 2008, 2013, Barra et al. 2014) without increasing corticosterone levels (Pacak et al. 1998) or affecting gonadotropin levels (Bernuci et al. 2013). The increase in ovarian NA after 28 days of chronic cold stress is related to alterations in follicular development, such as a decrease in secondary follicles and the appearance of follicles with a hypertrophic theca layer (Dorfman et al. 2003, Zangeneh et al. 2011, Park et al. 2012). These follicles with hyperthecosis produce high levels of androgens, and these hormonal changes are thought to be responsible for the formation of precystic follicles (i.e. follicles in the transition toward cysts) and ovarian cysts (Dissen et al. 2000, Paredes et al. 2011). When the exposure to cold stress is prolonged up to 8 weeks, the ovary still presents follicles with hyperthecosis, precystic follicles and even higher number of follicular cysts, which implies the development of a polycystic ovary phenotype in rat (Bernuci et al. 2013).

Although all of these data strongly suggest profound changes in ovarian follicular development, it is not known: (a) if these changes are associated with a decreased fertility of the rats as a consequence of the cold stress exposure and (b) which is the main neurotransmitter involved in these responses.

In addition to NA, there is also a cholinergic system in the ovary. This cholinergic system is local and associated with follicular granulosa cells (GC), which produce and store acetylcholine (ACh) (Fritz et al. 1999). Both in vivo and in vitro experiments showed that human GCs also express several muscarinic (MR) subtype receptors (Mayerhofer \& Fritz 2002, Cruz et al. 2015). Finally, the enzyme that degrades ACh, acetylcholinesterase (AChE), which hydrolyzes ACh to choline and acetate (Soreq \& Seidman 2001) was also characterized in rat ovary (Guraya et al. 1991). Recently, it was determined that the chronic, intraovarian administration of Huperzine A (Hup A), a drug inhibiting the catalytic activity of AChE and thereby elevates intraovarian $\mathrm{ACh}$, also increased follicular development. These rats when mated with fertile males presented increased implantation sites and number of live neonates (Urra et al. 2016).
The purpose of this work was to elucidate the consequences of cold stress on intraovarian NA and ACh, and to determine their relationship with structural and functional ovarian changes, namely ovarian follicular growth, atresia, ovulation, hormone production and fertility in the rat.

\section{Methods}

\section{Animals and experimental design}

A total number of 40 female Sprague-Dawley rats weighing 250-300 g were used. All animals were housed in a maintenance room at a temperature of $20^{\circ} \mathrm{C}$ with light-dark cycles $(12: 12 \mathrm{~h})$. The animals had food and water ad libitum. Twenty rats were divided in two groups of ten each. One group was exposed to cold stress for 28 days ( $3 \mathrm{~h}$ a day at $4^{\circ} \mathrm{C}, 5$ days a week) or (the other 10 rats) at room temperature (Control), after this period, rats were unilateral ovariectomized (ULO), removing the right ovary. Five ovaries were used to measure NA and ACh levels, the other five were fixed in Bouin's and cut in slices to do a morphometric analysis, which was performed to analyze the follicular development at the end of this period of exposure to stress. After unilateral ovariectomy, the remaining ovary increases in weight, size and number of follicular structures (compensatory ovarian hypertrophy). All these changes seem to represent general compensatory changes that do not have a greater implication for the results, because the differences were no longer evident when data are normalized by size (Chávez \& Domínguez 1994, Trkulja et al. 2004, MoralesLedesma et al. 2011). The groups of rats were examined by daily vaginal smears for estrous cycle regularity. Results are presented as the number of estrous cycles considering the following stage of the estrous cycle: proestrus (P), estrus (E), diestrus (D). Control rats presented a regular 4-day estrous activity (Hubscher et al. 2005, Paccola et al. 2013). A retrospective evaluation of the data from our animals show that all control rats show a range of $60-80 \%$ of estrous cycles (considering short cycles of 4 days and long cycles of 5 days). Considering this 20\% deviation between the length of the estrous cycles, we considered a normal behavior for the percentage found (Del Campo et al. 2019).

At the end of the procedure the rats were killed by decapitation, the ovaries and plasma were collected. All experimental procedures were approved by the Bioethics Committee of the Faculty of Chemistry and Pharmaceutical Sciences at the University of Chile 
(Protocol number: CBE2016-13) and complied with national guidelines (CONICYT Guide for the Care and Use of Laboratory Animals).

\section{Morphometric analysis}

Ovaries were fixed in Bouin's fluid, embedded in paraffin, cut into $6 \mu \mathrm{m}$ sections and stained with hematoxylin and eosin. Morphometric analyses of whole ovaries were done according to (Hirshfield \& Midgley 1978) with modification described in (Lara et al. 2000), using $n=4$ ovaries of a group exposed to 28 days of stress, and a further group exposed to 28 days stress followed by 28 days without stress and corresponding controls (five ovaries). All follicular structures were followed through all sections and counted in every fifth slice. Briefly, primordial follicles were those with one oocyte surrounded by flattened GCs. Primary follicles exhibited one layer of cubical GCs and secondary follicles had no antral cavity but two or more layers of GCs. Antral follicles were those with more than three healthy GC layers, the antrum and a clearly visible nucleus of the oocyte. Atretic follicles had more than 5\% of cells with pyknotic nuclei in the largest cross-section and exhibited shrinkage and an occasional breakdown of the germinal vesicle. Precystic follicles were large follicles with or without oocyte, containing four or five plicated layers of small, densely packed GCs surrounding a very large antrum with an apparently normal thecal compartment. Cystic follicles were devoid of oocytes and displayed a large antral cavity, a well-defined thecal cell layer and a thin (mostly monolayer) GC compartment containing apparently healthy cells (Lara et al. 2000). All these abnormal follicular structures were grouped as cystic structures.

\section{Fertility}

Ten adults rats were exposed to 28 days of cold stress ( $3 \mathrm{~h}$ a day at $4^{\circ} \mathrm{C}, 5$ days a week) and other ten were maintained at room temperature (control); after this period, the rats were unilaterally ovariectomized (ULO). Twenty-eight days post stress, they were mated with males of proven fertility to evaluate female fertility (rate in percentage between the number of rats that become pregnant and have an offspring and the rats that do not become pregnant).

\section{Number of offspring and implantations in uterine horns}

The rats were exposed to cold stress for 28 days ( $3 \mathrm{~h}$ a day at $4^{\circ} \mathrm{C}, 5$ days a week) or kept at room temperature (control), and after this period, rats were unilateral ovariectomized (ULO). After the 28 days post stress period, they were mated with males of proven fertility to quantify the number of offspring. Later, the mothers were killed on day 4 after delivery and will undergo surgery to expose the uterine horns, to examine the number of implants in the uterine horn and compare them with the number of offspring born.

\section{Quantification of intraovarian levels of ACh and acetylcholinesterase activity}

The ovary was homogenized in ten volumes of PBS in ice. ACh determination was performed in the homogenate by using the Amplex ${ }^{\circledR}$ Acetylcholine/Acetylcholinesterase Assay kit following the instructions recommended by the provider (Thermo Fisher). Briefly, ACh is hydrolyzed by AChE, the choline formed is then oxidized by choline oxidase, and the $\mathrm{H}_{2} \mathrm{O}_{2}$ resulting from this reaction, interacts with Amplex Red reagent (10-acetyl-3,7dihydroxyphenoxazine) in the presence of horseradish peroxidase to form the highly fluorescent resorufin. Results are expressed by the total amount of ACh in $\mu \mathrm{mol}$ per ovary. The minimal detectable value for AChE was $0.002 \mathrm{U} / \mathrm{mL}$ and for $\mathrm{ACh}$ was $0.3 \mu \mathrm{M}$, range $0.3 \mu \mathrm{M}$ to $100 \mu \mathrm{M}$ (Invitrogen).

\section{Quantification of intraovarian NA levels}

The ovary was homogenized in ten volumes of cold $0.2 \mathrm{~N}$-perchloric acid. Homogenate was centrifuged at $10,000 \boldsymbol{g}$ during $10 \mathrm{~min}$ and the supernatant used to determine NA. Quantification of NA levels was carried out using the Norepinephrine ELISA kit - Research ${ }^{\circledR}$ (Immusmol, Pessac, France). The minimal detectable value was $1.3 \mathrm{pg} / \mathrm{mL}$, range $0.2-32 \mathrm{ng} / \mathrm{mL}$. NA was extracted using a cis-diol-specific affinity gel, acylated and then derivatized enzymatically. The antigen was bound to the solid phase of the microtiter plate. The derivatized standards, controls and samples, as well as the solid-phase-bound analyte, compete for a fixed number of antiserum-binding sites. The antibody bound to the solid phase was detected using an anti-rabbit IgG-peroxidase conjugate and $\mathrm{TMB}$ as a substrate. The reaction is monitored at $450 \mathrm{~nm}$. Results are expressed by the total amount of NA in ng per ovary. The sensitivity was $2 \mathrm{pg} / \mathrm{mL}$, and the intra and interassay variability were 8.4 and $8.0 \%$, respectively. The crossreactivity found was $0.14 \%$ for adrenaline and $1.8 \%$ for dopamine. 


\section{Plasma levels of steroid hormones}

Plasma levels of steroid hormones testosterone, estradiol (E2) and progesterone (P4) were measured. Serum levels of testosterone, E2 and P4 were determined by EIA, following the manufacturer's instructions. Intraand interassay variations were less than $5 \%$ for E2, less than $6 \%$ for testosterone and less than $5 \%$ for P4. The minimal detectable values were $10 \mathrm{ng} / \mathrm{mL}$, $0.02 \mathrm{ng} / \mathrm{mL}$, and $0.1 \mathrm{ng} / \mathrm{mL}$, respectively (Alpco Diagnostic, Windham, NH, USA).

\section{Immunohistochemistry}

The method was described previously (Squicciarini et al. 2018). In brief, ovaries were fixed in Bouin's fluid, embedded in paraffin and cut at $6 \mu \mathrm{m}$ each (to perform the IHC techniques we used the central slices obtained from the middle section of all ovaries after they were used for morphometric analysis). Antigen recovery was done by microwave heating at $800 \mathrm{~W}$ for $10 \mathrm{~min}$ in buffer Tris/ EDTA pH 9.0, followed by wash steps (three times with PBS). Samples were incubated for $30 \mathrm{~min}$ in $3 \%$ hydrogen peroxide with $10 \%$ methanol to block endogenous peroxidases. The AChE monoclonal antibody ab183591 from Abcam RabMAb was used, which recognizes all AChE isoforms. Incubation was done for two days with 1:20 antibody solution in PBS in the presence of 5\% normal horse serum. On the third day, the tissue was washed in PBS and incubated with the secondary biotinylated antibody (1:50) for $2.5 \mathrm{~h}$. After incubating the samples with HRPstreptavidin, the ImmPACT VIP Peroxidase Substrate kit (Vector Laboratories) was used as a chromogen. As a negative control, we used antibody preabsorbed with the immunogenic peptide ab45700 from Abcam. In addition, a polyclonal antibody P-18 from Santa Cruz Biotechnology was used to examine $3 \beta$-HSD. The incubation was done overnight (dilution 1:500). Images were obtained using an Olympus light microscope (Olympus CX31) with Micrometrics SE Premium 4 software (ACCU-SCOPE, Inc., Commack, NY, USA).

\section{Statistical analysis}

The data are expressed as the mean \pm S.E.M. To examine statistical differences between two groups, we used two-tailed Student's $t$-test. To analyze differences between several groups, we used one-way ANOVA followed by a Newman-Keul's post-test. To analyze difference between proportions, we used chi-square test (Prism GraphPad).

\section{Results}

\section{Effect of cold stress on fertility}

The control rats presented normal estrous cycling activity during $60 \%$ of the time analyzed (Fig. 2A). The same pattern was found after 4 week of cold stress (Fig. 1, middle bar). Cyclicity in stressed rats observed during an extra 28 days (post stress period), was in contrast, significantly affected. A decrease to less of $40 \%$ the number of normal cycles during the period (Fig. 2, right bar) as compared to control rats became apparent. For the fertility study, we consider all rats including the rats without cycling activity. Figure $2 \mathrm{~B}$ shows the percentage of rats that remained not pregnant along with the pregnant rats of the control and stress groups. This result is translated into the fertility rate of both groups. Stressed rats decreased the fertility in 30\% compared with control rats group. There are no changes in the number of born pups (Fig. 2C) and implantation sites (Fig. 2D).

\section{Effect of cold stress on ovarian neurotransmitters}

We studied the changes in NA, ACh and AChE associated with the stress response, and the persistence of the changes after the stress procedure. Ovarian ACh and NA levels were increased significantly after 28 days of stress (Fig. 3A and C). Twenty-eight days after the end of the stress period, ACh content had returned to the control

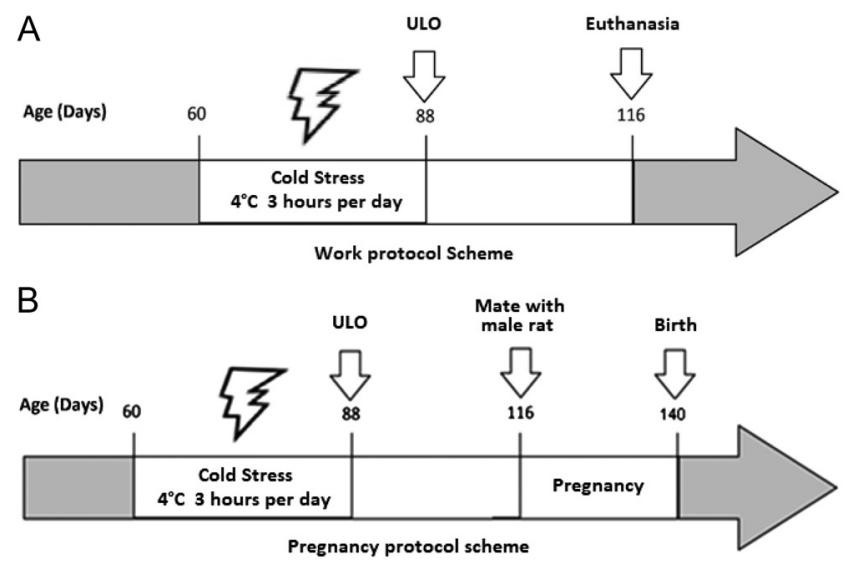

Figure 1

(A) Work protocol scheme. (B) Pregnancy protocol scheme. ULO, unilateral hemiovariectomy. (c) 2019 Society for Endocrinology Published by Bioscientifica Ltd.
Printed in Great Britain 

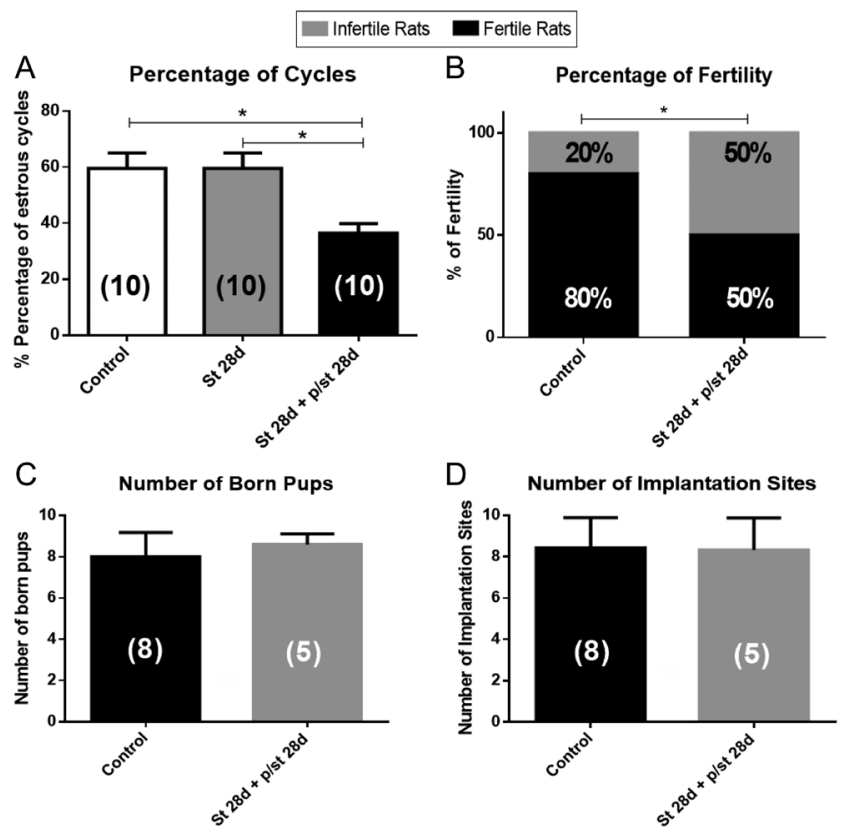

Figure 2

Estrous cycles expressed in percentage of cycles (A). 100\% correspond to the total theoretical number of cycles if the rat present 4-day estrous cycles ( 7 cycles corresponds to 28 days and thus to $100 \%$ ). Fertility of rats in percentage (rate in percentage between the number of rats that become pregnant and have an offspring and the rats that do not become pregnant). (B) The groups correspond to control (Control), 28 days cold stress exposure (St $28 \mathrm{~d}$ ) and 28 days cold stress exposure followed by 28 days period post stress (St $28 d+$ p/st $28 d$ ) $(n=10)$; mean \pm S.E.M. Number of born pups $(C)$ and number of implantation sites per rat found either in the uteri of controls or cold stress-exposed rats, 28 days after cessation of stress rats (D). The groups correspond to control (Control, $n=8$ ) and 28-day cold stress exposure followed by 28-day period post stress (St $28 d+$ p/st $28 d, n=5$ ). Mean \pm S.E.M.

values (Fig. 3A and C). The levels of AChE activity followed the same pattern as ACh (Fig. 3B).

\section{Effect of cold stress in follicular dynamics of ovary follicular development}

Because of the previously demonstrated changes in follicular dynamic after stress (Dorfman et al. 2003, Bernuci et al. 2008, 2013), we analyzed ovarian follicle composition at the end of the stress procedure and after the stress-free second period. Figure 4 shows representative images of rat ovaries of all groups (control, after the first and second period). The 4 weeks of chronic cold stress exposure did not induce obvious changes in ovarian morphology (Fig. 4B); yet, such changes appeared after the second 28 days post-stress period, when cysts were found (Fig. 4C). However, when we quantified the preantral and antral follicles population a decreased number of secondary follicles was noted after the stress
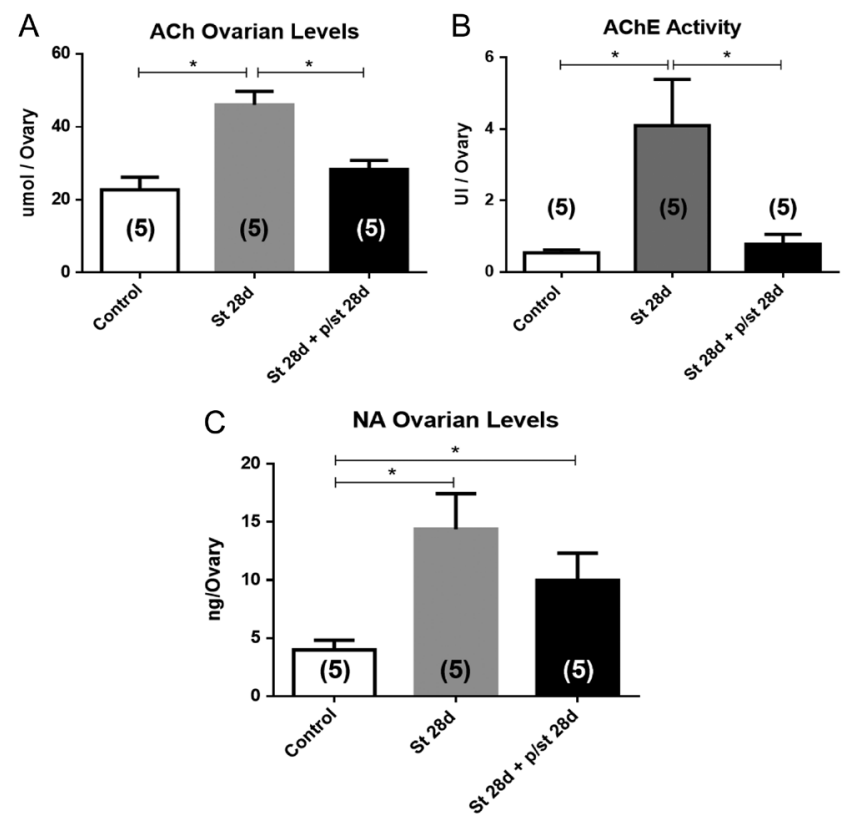

Figure 3

Acetylcholine ovarian levels (A), acetylcholinesterase activity (B) and noradrenaline ovarian levels (C). The groups correspond to control (Control), 28 days cold stress exposure (St $28 \mathrm{~d}$ ) and 28 days cold stress exposure followed by 28 days period post stress (St $28 d+p / s t 28 d$ ). $(n=5)$; Mean \pm S.E.M.

period (Fig. 5A). Furthermore, the proportions of healthy and atretic antral follicles changed and atretic antral follicles were increased immediately after cold stress exposure, and even more so 4 weeks after cessation of the cold stress exposure (Fig. 5B). In addition, at the end
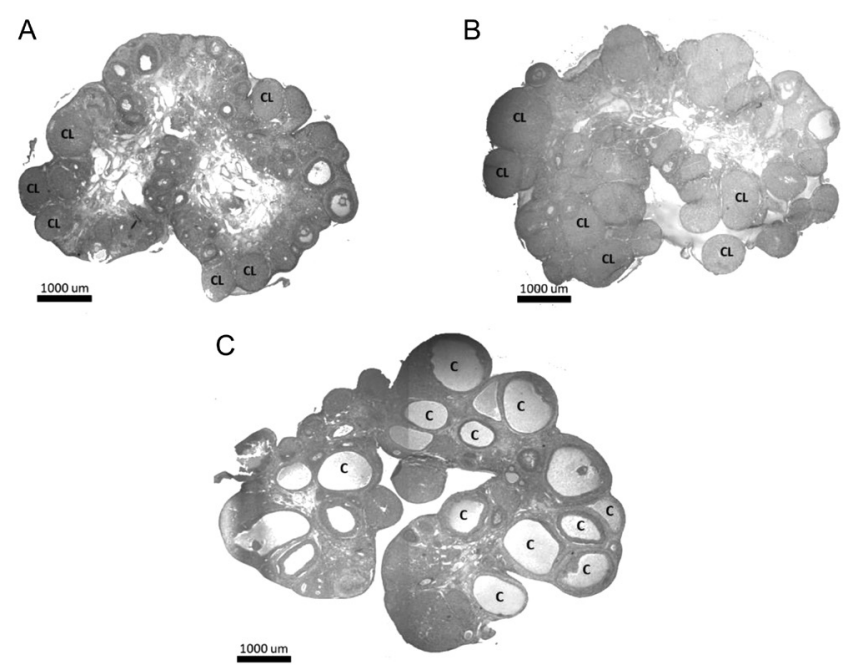

\section{Figure 4}

(A) Representative picture of a control ovary, (B) an ovary after 28 days of cold stress exposure and (C) an ovary, exposed to 28 days of cold stress followed by 28-day period post stress period. In the pictures corpora lutea $(\mathrm{CL})$ and cysts $(\mathrm{C})$ are marked. 

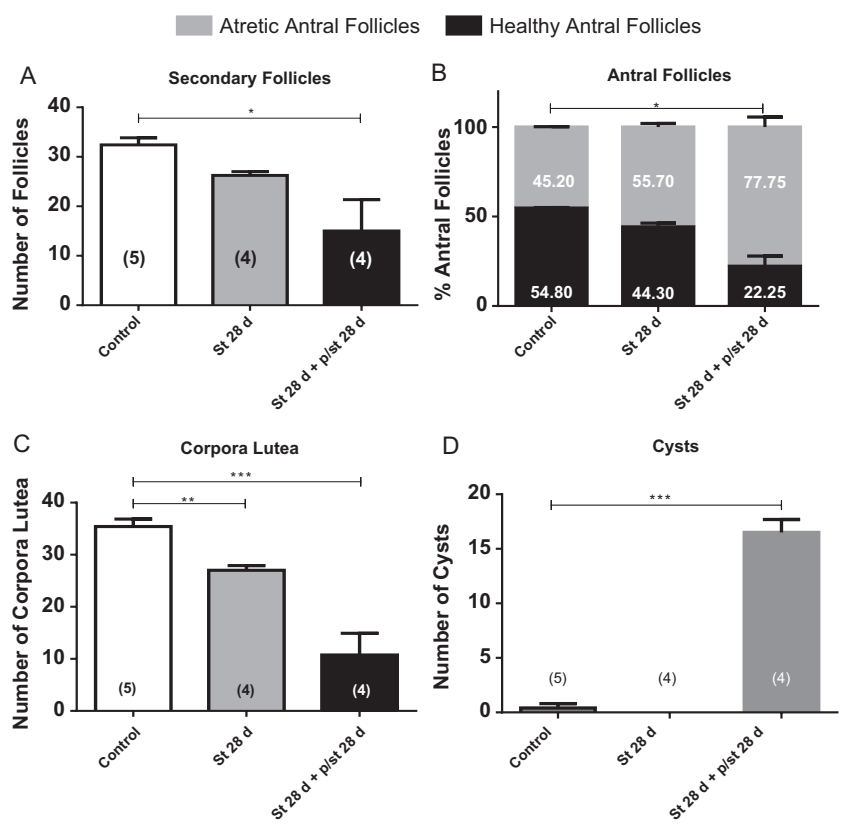

\section{Figure 5}

Secondary follicles (A), percentage of healthy and atretic antral follicles (B), corpora lutea (C) and cysts (D). The groups correspond to control (Control), 28 days cold stress exposure (St $28 \mathrm{~d}$ ) and 28 days cold stress exposure followed by 28 -day period post stress (St $28 \mathrm{~d}+\mathrm{p} / \mathrm{st} 28 \mathrm{~d}$ ). (Control group $n=5$, St 28 and St $28 \mathrm{~d}+$ p/st $28 \mathrm{~d} n=4$ ); mean \pm S.E.M.

of cold stress exposure, the number of corpora lutea was decreased and continued to decrease in the second period, i.e. 4 weeks after cold stress exposure (Fig. 5C). Cystic follicles were only found 4 weeks after the cessation of cold stress (Fig. 5C).

\section{Effect of cold stress in plasma levels of ovarian steroids}

The analysis of plasma levels of ovarian steroids at the end of the stress procedure and 28 days after its cessation show increased levels of testosterone at both time points (Fig. 6A). E2 plasma levels however were not modified during cold stress exposure but then were increased 28 days after its end (Fig. 6B). Regarding P4, there was a statistically significant and steady decrease up to the end of the complete experimental protocol (Fig. 6C).

\section{Effect of cold stress in the expression of $3 \beta-$ HSD and AChE}

No changes in the expression of $3 \beta$-HSD were observed in stress conditions (Fig. 7D and E). At the same time, no changes in the expression of AChE were observed in stress condition (Fig. 7A and B).

\section{Discussion}

The present results show that cold stress has severe consequences for the rat ovary and female fertility. Early changes include elevation of ovarian intraovarian NA and ACh levels. Upon cessation of stress, ACh returned to normal level, yet NA did not. This was associated with impaired follicular development and ovulation, resulting in the impairment of overall fertility. Cyst formation and hyperandrogenism were observed, which are in line with a PCO-like phenotype. The results show that decreased fertility in the female rat after cold stress exposure has a strong component of sympathetic nerve activity.

As previously described (Dorfman et al. 2003), the exposure to cold stress for 28 day increases NA ovarian levels. Cold stress condition increases the activity of noradrenergic neurons of the superior ovarian nerve pathway by central activation at the paraventricular nucleus (Jara et al. 2010), traveling through locus coeruleus neurons (Bernuci et al. 2008) to the sympathetic chain. That sympathetic nerves have an important role in the PCO phenotype has been described among others in experiments, in which chemical or surgical denervation of the ovarian nerve superior was evaluated (Espinoza et al. 2018). In the present study we also studied the changes

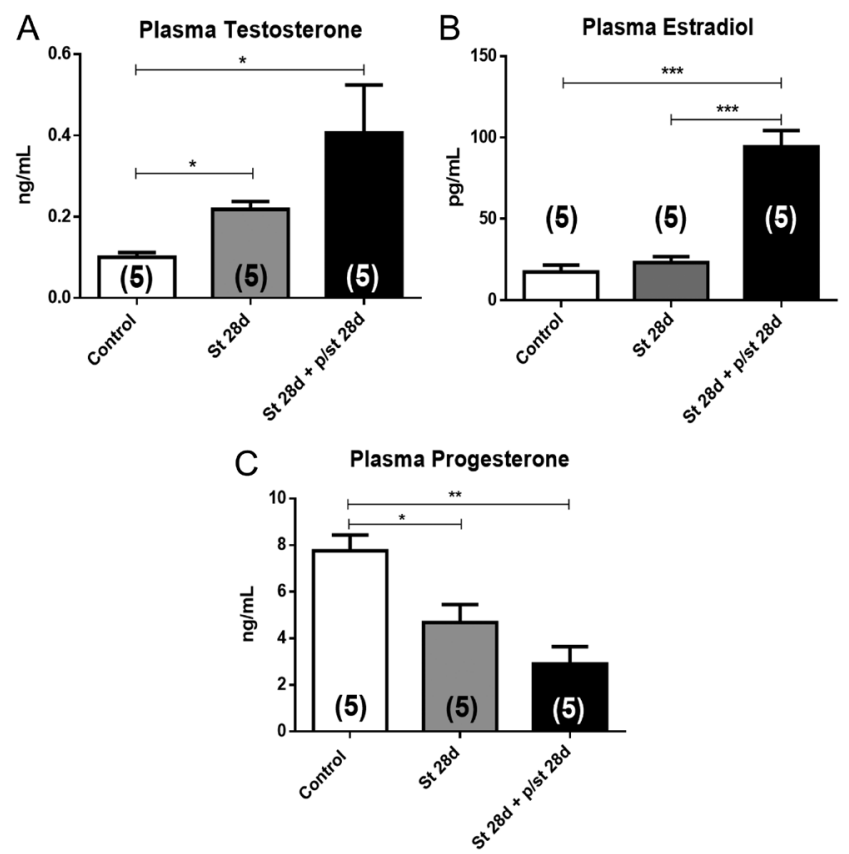

Figure 6

Plasma levels of testosterone (A), E2 (B) and P4 (C). The groups correspond to control (Control), 28 days cold stress exposure (St $28 \mathrm{~d}$ ) and 28 days cold stress exposure followed by 28 -day period post stress (St $28 \mathrm{~d}+$ p/st $28 \mathrm{~d}$ ). Testosterone/E2 ratios were not different between the groups. $(n=5)$; mean \pm S.E.M. 
CONTROL
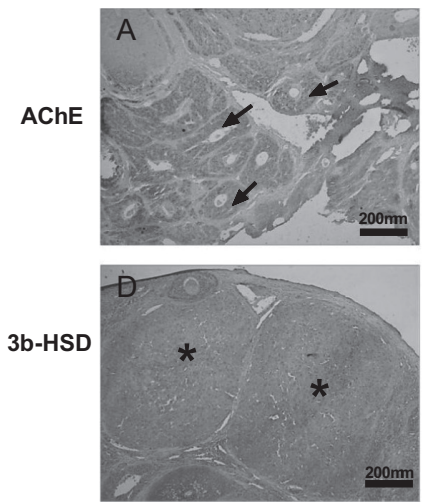

STRESS
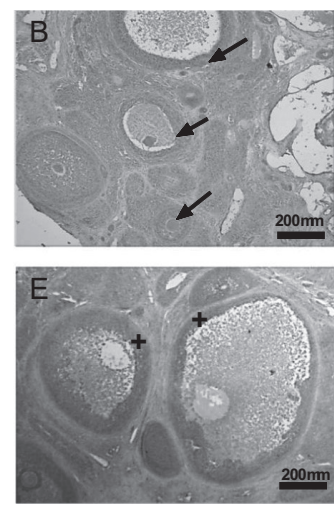

NEGATIVE CONTROL
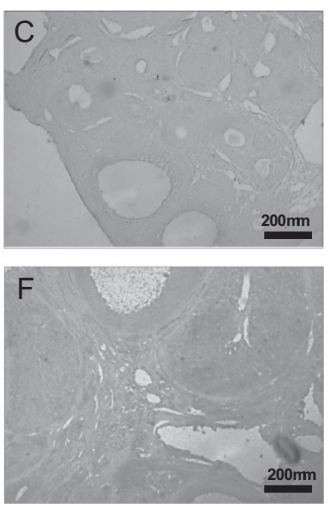

\section{Figure 7}

Immunohistochemical detection of AChE and $3 \beta$-HSD. (A) Representative picture of AChE detection in control ovary and (B) 28-day cold stress exposure followed by 28-day period post stress. (D) Representative picture for $3 \beta-H S D$ in a control ovary, and (E) after 28 days of cold stress exposure followed by 28-day period post stress. Negative controls (without primary antibody in case of $3 \beta-H S D$ and preabsorbed antibody in case of $A C h E$ ) are shown in (C) and (F). Arrows indicate staining of the GC of preantral follicles and antral follicles. Asterisks show positively stained luteinized GC and signs and crosses show staining in GC of antral follicles. of intraovarian ACh. The principal origin of intraovarian ACh are GCs of follicles, from which it is secreted to exert its trophic effects via the follicular muscarinic receptors (Mayerhofer \& Fritz 2002, Trkulja et al. 2004, Cruz et al. 2015). AChE is the principal degradation enzyme, and intraovarian ACh levels may principally be driven by an equilibrium between synthesis and degradation. The increase in ACh ovarian level found after 28 days of cold exposure is a local change, which manifests itself in ovarian follicles, and presumably may be an increase in intracellular, stored ACh (Gerendai et al. 2002). The activity of AChE, the principal enzyme that degrades $\mathrm{ACh}$, is like the ACh levels, increased in rats exposed to cold stress. A parallel increase in ACh content and AChE activity in response to stress has also been reported in brain areas, such as hippocampus and caudate nucleus (Imperato et al. 1991, Kaufer et al. 1998). If ACh is synthesized and stored inside the GC but not released, it is not accessible to the degrading enzyme. Only upon release into the extracellular space, it is either available to bind to the muscarinic or nicotinic receptors of the ovary (Soreq \& Seidman 2001) or becomes degraded by AChE. Trophic actions of bioactive ACh did become apparent in other non-stress conditions in vivo or ex vivo, previously. An increase in ovarian ACh levels were correlated with an increase in the size of cultured nonhuman primate follicles (Du et al. 2018), and elevated bioavailable ACh was responsible for the overall increased fertility and ovulation rate in the female rat, treated with an AChEblocker (Urra et al. 2016). Thus, even high levels of ACh - if primarily in intracellular stores - may not be able to exert trophic actions and thus are not able to interfere with or even reverse the changes produced by the increase of NA in the ovary.

It cannot be fully ruled out however that during the cold stress period at least some trophic actions of ACh did occur. The marked changes observed afterward may support such an assumption. After the 28-day cold stress and the following 28-day period post stress, the ACh levels decreased to control levels but NA were still higher than the control levels. These results suggest that the effect on the follicular development and the steroid hormones plasma levels are consequences of a dominant activation of the sympathetic system.

As previously described (Dorfman et al. 2003), we also found that exposure to cold stress decreased the number of secondary follicles after the first month of stress exposure and as Bernuci et al. (2013) described, the remaining 28 days after the exposure, there was an even more pronounced effect. It is interesting to note that during the first 28 days of exposure to cold stress, both NA and ACh levels were increased. Taking together these data and those of Urra et al. (2016) who described that the increase in ACh intraovarian levels (without increase in NA), increased the secondary follicles, corpora lutea and the fertility in the rat, implies that when both systems are activated, there is a predominance of sympathetic tone on the ovarian function. Furthermore, during the remaining 28 days after cold stress exposure, only the NA levels remained increased without an increase in ACh levels, which would explain why after the end of the stress period, the derangement on follicular development was even more pronounced. Changes in NA and ACh levels could explain the changes in number of corpora lutea, which already decreased during the exposure to cold stress and showed a further profound decrease after its cessation. In addition, cystic follicles were not found after the stress period but appeared after its cessation, that is when the noradrenergic tone predominates. An increase in the number of cystic structures and decrease in the number of healthy structures, such as secondary follicles and antral follicles, result in a decrease in the number of the ovarian compartments which contain GC. Thereby the number of cells capable of producing and storing 
ACh would decrease. To further clarify the behavior of the cholinergic ovarian system during stress, the behavior of choline acetyl transferase (ChAT), the enzyme that synthesizes $\mathrm{ACh}$ and its distribution in cysts remains to be studied.

Overall, the decrease in follicular growth dynamics, expressed as a decrease in the number of corpora lutea, indirectly imply a decrease in the number of ovulations but in parallel, there was an increase in ovarian cyst development, which produces alterations in ovarian function, associated with androgen hypersecretion (Barria et al. 1993, Domínguez \& Cruz-Morales 2011, Espinoza et al. 2018). In this regard, the increased serum levels of testosterone and E2 were previously reported to appear 4 weeks after exposure to cold stress (Morales-Ledesma et al. 2010, Bernuci et al. 2013) and have been associated with hyperthecosis, precystic and cystic follicles induced by stress (Barria et al. 1993, Domínguez \& Cruz-Morales 2011). Comparing the levels of testosterone and E2 between the stress group and the control group, we also found a positive correlation with the increase in the ovarian cysts. These data were reinforced with the aromatization index obtained by the quotient between testosterone and E2. We did not found differences between the groups, implying that this increase in E2 levels corresponds to an increase in the substrate available for aromatization (see legend Fig. 4). On the other hand, the decreased levels of $\mathrm{P} 4$ of the rats exposed to cold stress is in line with a decrease in ovulatory rate of the stress-exposed rats. Immunoreactivity for $3 \beta$-HSD was readily detectable in all groups (Fig. 7D and E). This enzyme is responsible for the production of $\mathrm{P} 4$, and its expression indicates that decreased P4 is not due to a general lack of enzyme expression, but rather to the numerical decrease in the number of corpora lutea. It is important to note that these effects on follicular development and the secretion of steroidal hormones could be parallel events to the well-known ovarian control of the hypothalamus pituitary axis, since the levels of gonadotropins are not altered by exposure to cold stress (Bernuci et al. 2013). This last observation could be reinforced because rats subjected to cold stress can - in a lower capacity - still have estrous cycles and ovulations, despite all the morphological and hormonal changes produced in the ovary and can get pregnant.

The data show a participation of both the sympathetic system and the ovarian cholinergic local system in the functional impairments induced by cold stress. They also explain how the disturbances induced by a chronic

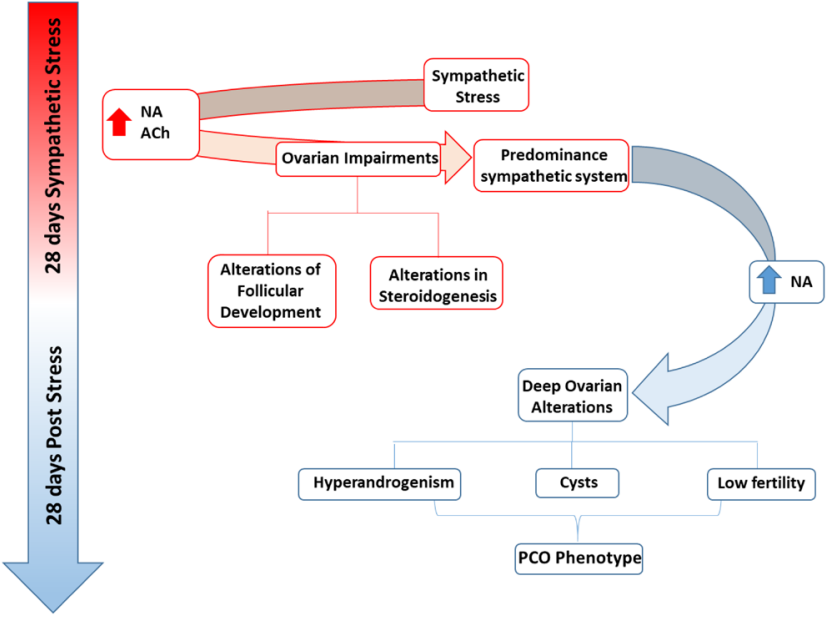

Figure 8

Temporal effect of cold stress on sympathetic and cholinergic systems. There is a predominance of the sympathetic system that continues after the end of the stress period. A full colour version of this figure is available at https://doi.org/10.1530/JOE-19-0125.

exposure to stress are translated in changes of follicular development and ovarian steroidogenesis that - as result of the balance of both activities - could induce changes in fertility of the rats (Fig. 8).

\section{Declaration of interest}

The authors declare that there is no conflict of interest that could be perceived as prejudicing the impartiality of the research reported.

\section{Funding}

This work was performed in partial fulfillment of the requirements of a PhD degree in Pharmacology to R R. This work was supported, in part, by Fondecyt grant 1170291 (to H E L). R R was also supported by a scholarship for Doctoral thesis support Conicyt $N^{\circ} 21170073$ and in part by DFG, MA1080/19-2 (to A M).

\section{Author contribution statement}

$\mathrm{R} R$ performed most of the experimental work with rats and biochemical analysis. F R conducted the experimental work and fertility test in the rats. A $M$ discussed the theoretical phases and experimental design of the work and contributed to draft the paper. $\mathrm{H} \mathrm{L}$ conceived the study, directed the work and drafted the paper. All authors participated in the writing of the manuscript and approved of the final version.

\section{References}

Aguado LI 2002 Role of the central and peripheral nervous system in the ovarian function. Microscopy Research and Technique 59 462-473. (https://doi.org/10.1002/jemt.10232) 
Barra R, Cruz G, Mayerhofer A, Paredes A \& Lara HE 2014 Maternal sympathetic stress impairs follicular development and puberty of the offspring. Reproduction 148 137-145. (https://doi.org/10.1530/ REP-14-0150)

Barria A, Leyton V, Ojeda SR \& Lara HE 1993 Ovarian steroidal response to gonadotropins and beta-adrenergic stimulation is enhanced in polycystic ovary syndrome: role of sympathetic innervation. Endocrinology 133 2696-2703. (https://doi.org/10.1210/ endo.133.6.8243293)

Bernuci MP, Szawka RE, Helena CV, Leite CM, Lara HE \& AnselmoFranci JA 2008 Locus coeruleus mediates cold stress-induced polycystic ovary in rats. Endocrinology 149 2907-2916. (https://doi. org/10.1210/en.2007-1254)

Bernuci MP, Leite CM, Barros P, Kalil B, Leoni GB, Del BiancoBorges B, Franci CR, Szawka RE, Lara HE \& Anselmo-Franci JA 2013 Transitory activation of the central and ovarian norepinephrine systems during cold stress-induced polycystic ovary in rats. Journal of Neuroendocrinology 25 23-33. (https://doi.org/10.1111/j.13652826.2012.02373.x)

Chávez R \& Domínguez R 1994 Participation of the superior ovarian nerve in the regulation of compensatory ovarian hypertrophy: the effects of its section performed on each day of the oestrous cycle. Journal of Endocrinology 140 197-201. (https://doi.org/10.1677/ joe.0.1400197)

Cruz ME, Flores A, Alvarado BE, Hernández CG, Zárate A, Chavira R, Cárdenas M, Arrieta-Cruz I \& Gutiérrez-Juárez R 2015 Ovulation requires the activation on proestrus of $\mathrm{M}_{1}$ muscarinic receptors in the left ovary. Endocrine 49 809-819. (https://doi.org/10.1007/s12020014-0524-3)

Del Campo M, Piquer B, Witherington J, Sridhar A \& Lara HE 2019 Effect of superior ovarian nerve and plexus nerve sympathetic denervation on ovarian-derived infertility provoked by estradiol exposure to rats. Frontiers in Physiology 10 349. (https://doi.org/10.3389/ fphys.2019.00349)

Dissen GA, Lara HE, Leyton V, Paredes A, Hill DF, Costa ME, MartinezSerrano A \& Ojeda SR 2000 Intraovarian excess of nerve growth factor increases androgen secretion and disrupts estrous cyclicity in the rat. Endocrinology 141 1073-1082. (https://doi.org/10.1210/ endo.141.3.7396)

Domínguez R \& Cruz-Morales S 2011 The ovarian innervation participates in the regulation of ovarian functions. Endocrinology and Metabolic Syndrome S4 001.

Dorfman M, Arancibia S, Fiedler JL \& Lara HE 2003 Chronic intermittent cold stress activates ovarian sympathetic nerves and modifies ovarian follicular development in the rat. Biology of Reproduction $\mathbf{6 8}$ 2038-2043. (https://doi.org/10.1095/biolreprod.102.008318)

Du Y, Bagnjuk K, Lawson MS, Xu J \& Mayerhofer A 2018 Acetylcholine and necroptosis are players in follicular development in primates. Scientific Reports 8 6166. (https://doi.org/10.1038/s41598-018-24661-z)

Espinoza JA, Alvarado W, Venegas B, Domínguez R \& Morales-Ledesma L 2018 Pharmacological sympathetic denervation prevents the development of polycystic ovarian syndrome in rats injected with estradiol valerate. Reproductive Biology and Endocrinology 1686 (https://doi.org/10.1186/s12958-018-0400-8)

Fritz S, Föhr KJ, Boddien S, Berg U, Brucker C \& Mayerhofer A 1999 Functional and molecular characterization of a muscarinic receptor type and evidence for expression of choline-acetyltransferase and vesicular acetylcholine transporter in human granulosa-luteal cells. Journal of Clinical Endocrinology and Metabolism 84 1744-1750. (https://doi.org/10.1210/jcem.84.5.5648)

Gerendai I, Kocsis K \& Halász B 2002 Supraspinal connections of the ovary: structural and functional aspects. Microscopy Research and Technique 59 474-483. (https://doi.org/10.1002/jemt.10225)

Guraya SS, Kaur P \& Sharma RK 1991 Histochemical and biochemical studies on esterase activity in the rat ovary. European Journal of Morphology 29 161-172.
Havelock JC, Rainey WE \& Carr BR 2004 Ovarian granulosa cell lines. Molecular and Cellular Endocrinology 228 67-78. (https://doi. org/10.1016/j.mce.2004.04.018)

Hirshfield AN \& Midgley AR 1978 Morphometric analysis of follicular development in the rat. Biology of Reproduction 19 597-605. (https:// doi.org/10.1095/biolreprod19.3.597)

Hubscher CH, Brooks DL \& Johnson JR 2005 A quantitative method for assessing stages of the rat estrous cycle. Biotechnic and Histochemistry 80 79-87. (https://doi.org/10.1080/10520290500138422)

Imperato A, Puglisi-Allegra S, Casolini P \& Angelucci L 1991 Changes in brain dopamine and acetylcholine release during and following stress are independent of the pituitary-adrenocortical axis. Brain Research 538 111-117. (https://doi.org/10.1016/0006-8993(91)90384-8)

Jara P, Rage F, Dorfman M, Grouselle D, Barra R, Arancibia S \& Lara HE 2010 Cold-induced glutamate release in vivo from the magnocellular region of the paraventricular nucleus is involved in ovarian sympathetic activation. Journal of Neuroendocrinology 22 979-986. (https://doi.org/10.1111/j.1365-2826.2010.02040.x)

Kaufer D, Friedman A, Seidman S \& Soreq H 1998 Acute stress facilitates long-lasting changes in cholinergic gene expression. Nature 393 373-377. (https://doi.org/10.1038/30741)

Lara HE, Dissen GA, Leyton V, Paredes A, Fuenzalida H, Fiedler JL \& Ojeda SR 2000 An increased intraovarian synthesis of nerve growth factor and its low affinity receptor is a principal component of steroid-induced polycystic ovary in the rat. Endocrinology 141 1059-1072. (https://doi.org/10.1210/endo.141.3.7395)

Lara HE, Dorfman M, Venegas M, Luza SM, Luna SL, Mayerhofer A, Guimaraes MA, Rosa E Silva AA \& Ramírez VD 2002 Changes in sympathetic nerve activity of the mammalian ovary during a normal estrous cycle and in polycystic ovary syndrome: studies on norepinephrine release. Microscopy Research and Technique 59 495-502. (https://doi.org/10.1002/jemt.10229)

Manni L, Cajander S, Lundeberg T, Naylor AS, Aloe L, Holmäng A, Jonsdottir IH \& Stener-Victorin E 2005 Effect of exercise on ovarian morphology and expression of nerve growth factor and alpha(1)- and beta(2)-adrenergic receptors in rats with steroid-induced polycystic ovaries. Journal of Neuroendocrinology 17 846-858. (https://doi. org/10.1111/j.1365-2826.2005.01378.x)

Mayerhofer A \& Fritz S 2002 Ovarian acetylcholine and muscarinic receptors: hints of a novel intrinsic ovarian regulatory system. Microscopy Research and Technique 59 503-508. (https://doi. org/10.1002/jemt.10228)

McGee EA \& Hsueh AJ 2000 Initial and cyclic recruitment of ovarian follicles. Endocrine Reviews 21 200-214. (https://doi.org/10.1210/ edrv.21.2.0394)

Morales-Ledesma L, Linares R, Rosas G, Morán C, Chavira R, Cárdenas M \& Domínguez R 2010 Unilateral sectioning of the superior ovarian nerve of rats with polycystic ovarian syndrome restores ovulation in the innervated ovary. Reproductive Biology and Endocrinology 899. (https://doi.org/10.1186/1477-7827-8-99)

Morales-Ledesma L, Ramírez DA, Vieyra E, Trujillo A, Chavira R, Cárdenas M \& Domínguez R 2011 Effects of acute unilateral ovariectomy to pre-pubertal rats on steroid hormones secretion and compensatory ovarian responses. Reproductive Biology and Endocrinology 9 41. (https://doi.org/10.1186/1477-7827-9-41)

Ojeda SR 2012. Female reproductive function. In Textbook of Endocrine Physiology, 6th ed. Ed WJ Kovacs. New York, NY, USA: Oxford University Press.

Pacak K, Palkovits M, Yadid G, Kvetnansky R, Kopin IJ \& Goldstein DS 1998 Heterogeneous neurochemical responses to different stressors: a test of Selye's doctrine of nonspecificity. American Journal of Physiology 275 R1247-R1255. (https://doi.org/10.1152/ ajpregu.1998.275.4.R1247)

Paccola CC, Resende CG, Stumpp T, Miraglia SM \& Cipriano I 2013 The rat estrous cycle revisited: a quantitative and qualitative analysis. Animal Reproduction 10 R677-R683. https://joe.bioscientifica.com https://doi.org/10.1530/JOE-19-0125 (c) 2019 Society for Endocrinology Published by Bioscientifica Ltd. Printed in Great Britain 
Paredes AH, Salvetti NR, Diaz AE, Dallard BE, Ortega HH \& Lara HE 2011 Sympathetic nerve activity in normal and cystic follicles from isolated bovine ovary: local effect of beta-adrenergic stimulation on steroid secretion. Reproductive Biology and Endocrinology 9 66. (https://doi. org/10.1186/1477-7827-9-66)

Park E, Cockrem JF, Han KH, Kim DH, Jung MH \& Chu JP 2012 Stressinduced activation of ovarian heat shock protein 90 in a rat model of polycystic ovary syndrome. Journal of Obstetrics and Gynaecology Research 38 396-407. (https://doi.org/10.1111/j.1447-0756.2011.01705.x)

Pastelín CF, Rosas NH, Morales-Ledesma L, Linares R, Domínguez R \& Morán C 2017 Anatomical organization and neural pathways of the ovarian plexus nerve in rats. Journal of Ovarian Research 1018. (https://doi.org/10.1186/s13048-017-0311-x)

Rosas G, Linares R, Ramírez DA, Vieyra E, Trujillo A, Domínguez R \& Morales-Ledesma L 2018 The neural signals of the superior ovarian nerve modulate in an asymmetric way the ovarian steroidogenic response to the vasoactive intestinal peptide. Frontiers in Physiology 9 1142. (https://doi.org/10.3389/fphys.2018.01142)

Soreq H \& Seidman S 2001 Acetylcholinesterase - new roles for an old actor. Nature Reviews: Neuroscience 2 294-302. (https://doi. org/10.1038/35067589)
Squicciarini V, Riquelme R, Wilsterman K, Bentley GE \& Lara HE 2018 Role of RFRP-3 in the development of cold stress-induced polycystic ovary phenotype in rats. Journal of Endocrinology 239 81-91. (https:// doi.org/10.1530/JOE-18-0357)

Trkulja V, Crljen-Manestar V, Banfic H \& Lackovic Z 2004 Involvement of the peripheral cholinergic muscarinic system in the compensatory ovarian hypertrophy in the rat. Experimental Biology and Medicine 229 793-805. (https://doi. org/10.1177/153537020422900812)

Uchida S 2015 Sympathetic regulation of estradiol secretion from the ovary. Autonomic Neuroscience: Basic and Clinical 187 27-35. (https:// doi.org/10.1016/j.autneu.2014.10.023)

Urra J, Blohberger J, Tiszavari M, Mayerhofer A \& Lara HE 2016 In vivo blockade of acetylcholinesterase increases intraovarian acetylcholine and enhances follicular development and fertility in the rat. Scientific Reports 6 30129. (https://doi.org/10.1038/ srep30129)

Zangeneh FZ, Abdollahi A, Tavassoli P \& Naghizadeh MM 2011 The effect of cold stress on polycystic ovary syndrome in rat: before and during modeling. Archives of Gynecology and Obstetrics 284 651-657. (https:// doi.org/10.1007/s00404-010-1711-y)

Received in final form 31 May 2019

Accepted 7 June 2019

Accepted Preprint published online 7 June 2019
(C) 2019 Society for Endocrinology Published by Bioscientifica Ltd. 